\title{
NUMERICAL COUPLING OF FLUID AND STRUCTURE IN CARDIAC FLOW AND DEVICES
}

\author{
Idit Avrahami \\ Post Doctorate fellow, \\ Aeronautics and Bioengineering, \\ California Institute of Technology, CA
}

\begin{abstract}
Numerical simulations are a powerful tool in investigation of flow and structure dynamics in biological systems and in the design of biomedical devices. Time-dependent fluid-structure interaction (FSI) problems in biological systems are often characterized by a periodic nature and relatively low Reynolds number. In order to solve the dynamics of the fluid and structure of coupled systems, different approaches may be used. Several parameters such as geometrical complexity, degree of displacement, convergence to steady periodicity, and the system stability may determine the coupling method. In the talk, four numerical studies of biological and implanted systems will be presented, each with a different FSI approach. The first study is of flow through mechanical heart valves, using finite-volume (FV) fluid solver coupled with an external structural solver using a weak coupling scheme for large displacements. The second study is of flow inside a pulsatile ventricular assist device with FV fluid solver coupled with finite-element (FE) structure solver using a strong staggered coupling assuming small displacements. The third study is of flow through vulnerable plaque in the coronary arteries, with FE solvers for both the fluid and structure domains, using a fully-coupled iterative scheme assuming small displacements. The fourth simulation is of an impedance pump using a direct FE coupling method for large displacements. In addition to the methodology, the applicative design and hemodynamic aspects of the cases will be discussed, including washout properties and risk for thrombosis. The results obtained from the studies will be compared to experimental analyses.
\end{abstract}

